\title{
PERSISTENCIA DE PRODUCTORES FAMILIARES EN EL SUDOESTE BONAERENSE. UN ESTUDIO DE CASOS PARA SU CARACTERIZACIÓN
} Persistence of FAMILY PRODUCERS IN SOUTHWEST BONAERENSE. A CASE STUDY FOR ITS CHARACTERIZATION

Fecha recepción: 8 de septiembre de 2020 / fecha aceptación: 29 de diciembre de 2020

Sofía Hang ${ }^{1}$ y Edgardo Gonzalez ${ }^{2}$

Cómo citar este artículo:

Hang, S. y Gonzalez, E. (2020). Persistencia de productores familiares en el sudoeste bonaerense. Un estudio de casos para su caracterización. Revista Pensamiento y Acción Interdisciplinaria, 6(2), 70-86.

http://doi.org/10.29035/pai.6.2.70

\section{Resumen}

Las transformaciones acaecidas en las últimas décadas en las producciones agropecuarias han generado modificaciones en la estructura agraria argentina y con ellas cambios en los sujetos agrarios, aún en aquellos que han mostrado capacidad de resistir la tendencia a abandonar la actividad.

Estos sujetos agrarios intervienen en los territorios con diferentes estrategias productivas. En este sentido, existen múltiples dimensiones que permiten abordar los diversos grupos sociales que se encuentran en la estructura agraria. En este trabajo analizaremos algunas de ellas (tipos, formas de producción, vínculos con la tecnología, mercado, pluriactividad, entre otras) para acercarnos a una caracterización de estos sujetos.

Por este motivo, el presente trabajo tiene como objetivo principal la caracterización de los productores familiares del sudoeste bonaerense, en particular de los Municipios de Adolfo Alsina y Puan en su vinculación con el territorio.

Por otro lado, también se propone analizar si esta caracterización se adecúa a lo expresado en la Ley de Reparación Histórica de la Agricultura Familiar, Campesina e Indígena N²7.118 del estado argentino.

El abordaje metodológico que se propone se basa en un enfoque principalmente cualitativo. Se combinan distintas técnicas cualitativas y constará de relevamientos de fuentes primarias y secundarias. Especialmente se plantea el estudio de casos y como herramientas de relevamiento entrevistas semiestructuradas, análisis de documentos y datos cuantitativos como encuestas, datos numéricos ya existentes y datos de los Censos Agropecuarios Nacionales de 1988, 2002 y 2018.

Palabras clave: productores familiares, sudoeste bonaerense, territorio

\footnotetext{
1 Argentina, abogada, doctoranda, CONICET-INTA-UNLP, La Plata, Argentina. Correo electrónico: sofihang@hotmail.com
} 2 Argentino, abogado, INTA-UNLP, Bordenave, Argentina. Correo electrónico: gonzalez.edgardo@inta.gob.ar 


\begin{abstract}
The transformations in agricultural production in the last decades generated modifications in the Argentine agrarian structure and with those changes the agrarian subjects also changed, even in those who have shown the ability to resist the tendency to abandon the activity.

These agrarian subjects intervene in the territories with different productive strategies. In this sense, there are multiple dimensions that allow approaching to all social groups found in the agrarian structure. In this work we will analyze some of them (types, forms of production, links with technology, market, multiple activities, etc.) to get closer to a characterization of these subjects.

For this reason, the main objective of this work is to characterize the family producers of the southwest of Buenos Aires, in particular of the Municipalities of Adolfo Alsina and Puan in their relationship with the territory.

On the other hand, also analyze if this characterization is adapted to what is expressed in the Law of Historical Reparation of Family Agriculture, Peasant and Indigenous No. 27,118 of the Argentine.

The methodological approach that is proposed is qualitative. Different qualitative techniques are combined and will consist of surveys of primary and secondary sources. In particular, the study of cases and semi-structured interviews, document analysis and quantitative data such as surveys, existing numerical data and data from the National Agricultural Censuses of 1988, 2002 and 2018.
\end{abstract}

Keywords: family producers, southwest of Buenos Aires, territory

\title{
Introducción
}

Las transformaciones acaecidas en las últimas décadas en las producciones agropecuarias han generado modificaciones en la estructura agraria argentina y con ella en los sujetos agrarios en las últimas décadas.

Los sujetos agrarios bajo análisis intervienen en los territorios con diferentes estrategias de acuerdo a sus determinaciones de vida, ya sea por elección propia o por entender que es la única posible de llevar adelante. En este sentido, existen múltiples dimensiones que permiten abordar los diversos grupos sociales que se encuentran en la estructura agraria. En este trabajo analizaremos algunas de ellas (tipos, formas de producción, vínculos con la tecnología, mercado, pluriactividad, entre otras) para acercarnos a una caracterización de estos sujetos.

Esta caracterización permitirá determinar si nos encontramos ante un sujeto que persiste y permanece en el territorio sin cambios, o si, al contrario, si estas estrategias que implementan, nos permiten denominarlos como nuevos sujetos agropecuarios. A su vez poder caracterizar si pertenecen, o no, a la categoría que se denomina Agricultura Familiar.

El trabajo tiene como objeto de estudio una selección de casos de productores agropecuarios familiares de los Municipios de Adolfo Alsina y Puan, ubicados en el sudoeste de la Provincia de Buenos Aires, Argentina. 
Persistencia de productores familiares en el sudoeste bonaerense. Un estudio de casos para su caracterización por Sofía Hang y Edgardo Gabriel Gonzalez

Estos partidos están comprendidos en esta región que es conformada en su integración por los municipios de: Guamaní, Adolfo Alsina, Coronel Suárez, Coronel Pringles, Coronel Dorrego, Saavedra, Tornquist, Puan, Coronel Rosales, Bahía Blanca, Villarino y Patagones (regionalización que utiliza la ley de plan de desarrollo del sudoeste bonaerense № 13.647 del año 2007 de la Provincia de Buenos Aires). Estos 12 municipios, de un total de 135 en la provincia, abarcan un territorio muy extenso, con muy poca población residente. Se caracteriza por la constitución de pueblos pequeños, algunas ciudades intermedias y solo una ciudad de envergadura: Bahía Blanca.

El sudoeste bonaerense constituye un recorte territorial interesante por haber estado atravesado históricamente por la constitución de grandes extensiones de tierra, procesos de colonización y planes de transformación agraria. Si bien en las últimas décadas se producen transformaciones a la luz de los cambios globales, se observa una permanencia de actores de la agricultura familiar extensivos ${ }^{3}$ (López Castro, 2012).

De este modo, Balsa y López Castro (2011, p.21) afirman que en el sudoeste de la Provincia de Buenos Aires: "si bien el contexto general de las últimas décadas ha resultado particularmente adverso para la producción familiar, existe un porcentaje relativamente importante de unidades productivas que lograron sostener su actividad bajo una forma de organización familiar o cercana a ella".

Es una zona relativamente poco explorada desde el punto de vista académico, en general por considerarse una "zona marginal" de la región pampeana, ya que, como se establece en los fundamentos de la ley № 13.647: "no es pampa húmeda, sino que forma parte de las regiones semiárida, árida y subhúmeda-seca del país, poseyendo características climáticas y edáficas que la diferencian del resto de la Provincia en cuanto a sus potencialidades y limitantes productivas primarias, y por lo tanto también, en cuanto a las mencionadas ventajas comparativas, claramente inferiores al resto de la Provincia".

Roberto Bustos Cara, en referencia a esta ley entiende que: "El sudoeste de la provincia de Buenos Aires (...) promovió la conciencia de la necesidad de formalización de un mecanismo de gestión del territorio rural que asegure la formulación de políticas diferenciales adaptadas a las condiciones propias; surge así a nivel provincial, la ley $N^{\circ} 13.647$ de creación del Plan de Desarrollo del Sudoeste de Buenos Aires" (Bustos Cara, 2010, p. 326).

En este sentido la funcionalidad de una ley específica para este recorte territorial tiene que ver con promocionar el arraigo de los habitantes del lugar, generar oportunidades de trabajo y fomentar el desarrollo local. En este marco

3 Se agrega nota personal. Producción extensivos por contraposición a intensivos. Son sistemas productivos vinculados predominantemente a la actividad pecuaria y agrícola, que se dan en grandes extensiones de tierra donde la productividad por hectárea no es tan alta como en las producciones intensivas, utilizando los bienes comunes de la zona y poca mano de obra. 
la actividad agraria es ampliamente predominante, por ello la necesidad de la aplicación de esta legislación en las políticas públicas específicas para este sector productivo.

\section{Marco teórico}

La Agricultura Familiar es entendida como un estilo de vida en el que la unidad doméstica y la unidad productiva se encuentran integradas. Son familias que producen tanto para el consumo propio como para la comercialización y la fuerza de trabajo es aportada en principio por la misma familia con muy poca mano de obra asalariada. Los medios de producción suelen ser de propiedad de las familias, aunque la tierra muchas veces no lo es (conceptualización general en base a lo establecido por la REAF ${ }^{4}$, el Estado Argentino y gran parte de organizaciones del sector).

Soverna, Tsakoumagkos y Paz (2008) plantean las diferencias entre dos definiciones cerradas y que consideran "oficiales" de agricultura familiar. Por un lado, presentan la definición del Programa de Desarrollo de Pequeños Productores Agropecuarios $^{5}$ (PROINDER) y por el otro la definición del Foro Nacional de la Agricultura Familiar (FONAF) ${ }^{6}$ y proponen la necesidad de generar nuevas definiciones y ponen en cuestión la utilidad de las anteriores.

La bibliografía vinculada a la temática, acuerda que tanto las características propias de los productores familiares como el escenario agrario pampeano en general han cambiado y se han transformado a lo largo del siglo XX (Balsa \& López Castro, 2011).

La ley nacional de Reparación Histórica de la Agricultura Familiar, Campesina e Indígena $\mathrm{N}^{\circ} 27.118$, sancionada en diciembre de 2014, describe a los agricultores y agricultoras familiares en su art. 5 como "aquel que lleva adelante actividades productivas agrícolas, pecuarias, forestal, pesquera y acuícola en el medio rural y reúne los siguientes requisitos:

a) La gestión del emprendimiento productivo es ejercida directamente por el productor y/o algún miembro de su familia;

b) Es propietario de la totalidad o de parte de los medios de producción;

4 REAF: Reunión Especializada de Agricultura Familiar del MERCOSUR. es un espacio de encuentro entre productores familiares, organizaciones e instituciones rurales de la región. Se creó en el año 2004 con el fin de crear políticas públicas regionales para la agricultura familiar.

5 “En el año 2006 el Programa de Desarrollo de Pequeños Productores Agropecuarios (PROINDER) de la SAGPyA, propició la necesidad de definir y cuantificar la pequeña producción agropecuaria en la Argentina en base a información del Censo Nacional Agropecuario (2002), y para ello convocó al IICA que armó un grupo ad hoc y produjo un documento3 en el que se adopta una definición de pequeños productores que por su amplitud puede asimilarse a la de agricultura familiar (AF). Allí se identifican tres tipos de pequeños productores básicamente diferenciados por la escala de las explotaciones agropecuarias -EAP- (tierra y/o capital) con que cuentan y, considerando diferencias regionales, se estima el peso de estos grupos en la producción y en el empleo" (Soverna, Tsakoumagkos y Paz, 2008, p. 5).

6 EI FONAF (2006), propone una definición cualitativa sobre la Agricultura Familiar, considerándola como una forma de vida y una cuestión cultural. 
c) Los requerimientos del trabajo son cubiertos principalmente por la mano de obra familiar y/o con aportes complementarios de asalariados;

d) La familia del agricultor y agricultora reside en el campo o en la localidad más próxima a él;

e) Tener como ingreso económico principal de su familia la actividad agropecuaria de su establecimiento;

f) Los pequeños productores, minifundistas, campesinos, chacareros, colonos, medieros, pescadores artesanales, productor familiar $y$, también los campesinos y productores rurales sin tierra, los productores periurbanos y las comunidades de pueblos originarios comprendidos en los incisos a), b), c), d) y e).

Javier Balsa (2012) describe a las unidades familiares arquetípicas, como aquellas en que las que la familia conforma un equipo de trabajo que se preocupa por la conservación del patrimonio familiar y forman un proyecto de vida vinculado a la actividad agropecuaria y con un cierto modo de vida rural deseable.

Respecto a estas familias, resulta interesante la idea que desarrollan Balsa y López Castro (2011, p. 2): "En general suele identificarse a las personas que llevan adelante la actividad como "productores", dando a la caracterización un sesgo masculino e individual, cuando la referencia a la familia debería incluir a los miembros de ambos géneros y a más de una persona (al equipo de trabajo). Es por esto que proponemos hablar de "familias productoras" en vez de "productores familiares", tratando de dar cuenta de la incidencia en la dinámica productiva y familiar del conjunto de sus miembros y de contrarrestar la tendencia a reproducir esquemas de poder establecidos, que se reflejan a nivel discursivo (aún de manera poco consciente)".

En este sentido, Balsa y López Castro (2011, p. 25) entienden que "las estrategias de estas familias son motivadas, tanto por los objetivos económicos que siguen la lógica capitalista en que se encuentran insertos, como por objetivos de satisfacción personal y familiar, de continuidad de tradiciones productivas y conservación de un legado a través de las generaciones. La preservación del patrimonio familiar y de un legado que traspasar a las generaciones más jóvenes aparece como un motivo de peso en la conformación de las estrategias de estas familias".

Balsa (2012), explica que en la agricultura familiar no se explota trabajo asalariado, sino que la familia conforma un equipo de trabajo con una racionalidad particular propia de la conjunción de la integración entre unidad productiva y doméstica, el rol que juega en la dinámica productiva-familiar la conservación del patrimonio familiar y, por último, la existencia de un proyecto de vida vinculado a la actividad agropecuaria. 
En relación a la no explotación de trabajo asalariado, otros autores como Azcuy Ameghino y Martínez Dougnac (2009, 2014), entienden que siguen siendo familiares aquellas unidades en las que el trabajo, o parte del mismo, está a cargo de asalariados, debido a que la gestión está en manos de los miembros de la familia.

La familia como equipo de trabajo, no implica que todos los miembros de la familia deban estar necesariamente involucrados en las tareas diarias en la explotación, sino que al menos un grupo de los integrantes lo esté.

Otro dato a destacar es que, en general, las familias viven y trabajan en el mismo predio, combinando la actividad agropecuaria con la satisfacción de las necesidades de consumo de la misma. También sucede que vivan en pueblos o ciudades cercanas, que les permitan concurrir diariamente al establecimiento productivo.

Lo que se demuestra a raíz del análisis bibliográfico (teórico y normativo) vinculado a la temática es que no existe un único modo de conceptualizar a estos sujetos, pero sí existen ciertos requisitos mínimos que deben estar presentes, como la dirección familiar y el aporte de mano de obra familiar. De esta multiplicidad de abordajes y caracterizaciones, surge la necesidad de esta investigación.

De este modo, Albadalejo y Cittadini (2017), sobre esta temática y heterogeneidad de sujetos que se encuentran en el territorio pampeano describen: "Descubrimos un personaje todavía anclado en el territorio local, notables agrarios de larga data con capacidad de transformarse en nuevos notables tanto en zonas de sojización como de ganadería. Probablemente este productor es factor de estabilidad de la actividad y del territorio si se lo compara con la falta de anclaje local que tienen en principio los actores más típicos del agribusiness. Por contraste con esas dos categorías que intentan hablar por él sin lograr representarlo (la agricultura familiar y el agribusiness), lo llamamos a este personaje el «productor moderno convencional». Nos hace de repente entender que nadie tiene el «monopolio de la modernización» y que las categorías de agricultura familiar y agribusiness representan dos formas posibles de modernidad, pudiendo existir otras más" (Albadalejo \& Cittadini, 2017, p. 31).

Es interesante este planteo ya que nos permite ampliar la mirada sobre el comportamiento general de todos los sujetos de este sector productivo en el territorio y este chacarero muy capitalizado o pequeño empresario agropecuario es parte del territorio, compartiendo con la agricultura familiar el anclaje al territorio, que hace que esté integrado a su desarrollo, sea parte de las instituciones locales y le preocupe la permanencia de los habitantes en el lugar.

En este sentido, Roberto Bustos Cara amplía estos enfoques, entendiendo que: "Tres formas de ruralidad parecen confrontarse en los espacios rurales: el mundo del agronegocio y la producción orientada a la exportación, asociada a un 
carácter empresario y un sistema financiero y técnico sofisticado; la agricultura familiar que reúne un amplio grupo de categorías y sistemas productivos que van desde el típico chacarero o productor capitalizado hasta los niveles de subsistencia y, por último, un sector que puede contenerse en el concepto de neorrural, que encierra numerosas formas de reincorporación de actividades y población de origen urbano al ámbito rural" (Bustos Cara, 2010, p. 323).

Tomando las categorías utilizadas por Bustos Cara (2010) y como un primer acercamiento a los sujetos con los que se trabajará, podemos englobarlos dentro de la de "agricultura familiar, grupo que reúne categorías y sistemas productivos que van desde el típico chacarero o productor capitalizado hasta los niveles de subsistencia...".

El objetivo principal que se plantea esta investigación es la caracterización de los productores familiares del sudoeste bonaerense, en particular de los Municipios de Adolfo Alsina y Puan en su vinculación con el territorio.

Por otro lado, como segundo objetivo se propone analizar si estos productores caracterizados de tal modo quedan contemplados en la Ley Nacional de Reparación Histórica de la Agricultura Familiar, Campesina e Indígena N²7.118.

\section{Metodología}

El abordaje metodológico propuesto se basa en un enfoque principalmente cualitativo (Martínez Carazo, 2006), en el que se combinan distintas técnicas cualitativas y consta de relevamientos de fuentes primarias y secundarias (documentos, resultados de encuestas, datos censales, registros de archivos, entre otros).

A su vez, se plantea una investigación diseñada desde la triangulación (Benavides \& Gómez, 2005). Esta triangulación se da en la relación y análisis del relevamiento de fuentes cuantitativas (datos censales) y cualitativas (entrevistas). Este método resulta adecuado ya que "no sólo sirve para validar la información, sino que se utiliza para ampliar y profundizar su comprensión" (Benavides \& Gómez, 2005, p.120).

Comoherramientas de relevamiento, seutilizan entrevistas semiestructuradas, análisis de documentos y datos cuantitativos de los Censos Agropecuarios Nacionales de 1988, 2002 y los datos existentes del censo del año 2018.

Los entrevistados se eligieron de acuerdo a diferentes parámetros: edad, género, lugar de residencia, diferencias en sus estrategias productivas y en su composición familiar. Las entrevistas fueron en profundidad y personales, entendiendo que es la mejor manera de indagar adecuadamente para estos objetivos. 
A su vez, cada uno de ellos, en mayor o menor medida, tiene una vinculación con los agentes locales del Instituto Nacional de Tecnología Agropecuaria (INTA). Como trabajadores de la institución, esto facilitó el contacto con los entrevistados.

En relación a los entrevistados y los parámetros elegidos, se entrevistaron sólo hombres ya que en un primer acercamiento al territorio no se tuvo contacto con mujeres productoras, sin embargo, al momento de realizar las entrevistas en los campos hubo participación de mujeres de las familias.

De acuerdo con el conocimiento que se tiene de la región bajo estudio, estos sujetos permiten ser identificados dentro de las categorías mencionadas. Se recuerda que no se busca, a través de los sujetos seleccionados, agotar la representatividad de los productores agropecuarios familiares del sudoeste bonaerense.

\section{Entrevistas y sujetos entrevistados}

Como se describió en el apartado metodológico, esta investigación se basa en un estudio cualitativo a partir de una serie de entrevistas. Los productores, cinco en total, fueron entrevistados en noviembre de 2019 en los municipios de Adolfo Alsina y Puan.

Por la vinculación con el INTA, las dos entrevistas del municipio de Adolfo Alsina se hicieron en la agencia local de extensión de la Institución en la localidad de Carhué. Las otras tres entrevistas se realizaron en los campos de los productores en el partido de Puan, una en la localidad de Bordenave, otra en Villa Iris y una en Darregueira.

Se analizaron seis variables: composición familiar, cantidad de tierra de la explotación agropecuaria, actividad que desempeñan, propiedad de maquinaria, contratación de mano de obra extrafamiliar y la pluriactividad.

Las cinco entrevistas se hicieron a productores hombres, pero en los tres casos de Puan estuvieron presentes mujeres (familiares) que de diversas formas se vinculan con la actividad productiva y que aportaron datos y comentarios en las entrevistas.

Todos los entrevistados son mayores de 40 años y se dedican a la producción agropecuaria. En todos los casos accedieron a la tierra a través de una herencia familiar. Es decir que viene con una vinculación intergeneracional con la actividad

7 El Instituto Nacional de Tecnología Agropecuaria un organismo estatal descentralizado con autarquía operativa y financiera, dependiente del Ministerio de Agricultura Ganadería y Pesca de la Nación. Fue creado en 1956 y desde entonces desarrolla acciones de investigación e innovación tecnológica en las cadenas de valor, regiones y territorios para el desarrollo rural sustentable del país. Trabaja de forma interinstitucional e interdisciplinaria para generar conocimientos y tecnologías y ponerlos al servicio del sector a través de sus sistemas de extensión, información y comunicación. 
agraria. Todos trabajan la totalidad de la tierra propia y en dos casos, además, arriendan tierra.

En cuatro casos la explotación familiar la llevan adelante los padres con sus hijos, en el caso restante sólo lo hace el matrimonio y un hijo que colabora esporádicamente en las tareas diarias. En dos casos hay pluriactividad y en los otros tres no la hay.

Cada uno de los casos realiza estrategias productivas diversas y sólo una familia tiene un empleado permanente, el resto contrata para tareas específicas en momentos determinados del ciclo productivo.

En relación a la maquinaria, cuatro productores tienen maquinaria propia y uno no. De los que sí tienen, dos tienen toda la maquinaria necesaria para su actividad y dos no, por lo que para la cosecha contratan el servicio a un tercero. Es también característica de la agricultura familiar tener sus propias herramientas de trabajo, pero por las características de las producciones extensivas sucede que en ocasiones resulta muy costoso adquirir determinada maquinaria, como lo es la cosechadora, por lo que se terceriza dicha actividad. Se puede destacar también que los dos casos con mayor cantidad de tierra trabajada tienen mayor cantidad de herramientas y de un tamaño mayor que el resto de los entrevistados. 
Aquí se presenta un cuadro que recoge el análisis anterior:

\begin{tabular}{|c|c|c|c|c|c|c|}
\hline 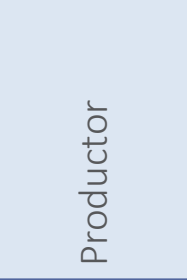 & $\begin{array}{l}0 \\
0 \\
0 \\
0 \\
0 \\
0 \\
\frac{0}{\square} \\
\frac{1}{2} \\
\frac{1}{0} \\
0\end{array}$ & 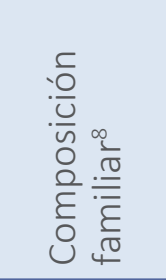 & 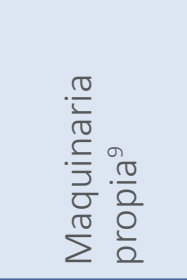 & 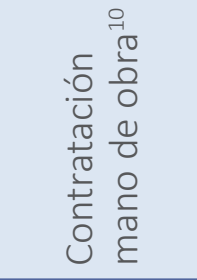 & 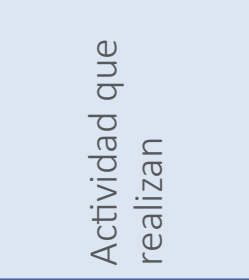 & $\begin{array}{l}7 \\
\frac{7}{0} \\
\frac{0}{0} \\
\cdot \frac{0}{2} \\
\frac{7}{U} \\
\cdot \frac{0}{2} \\
\frac{\partial}{2}\end{array}$ \\
\hline $\begin{array}{l}1 \text { (Carhué, } \\
\text { Adolfo Alsina) }\end{array}$ & $\begin{array}{l}66 \text { has. en } \\
\text { propiedad }\end{array}$ & $\begin{array}{l}\text { Él, su esposa, } \\
\text { su hija, yerno } \\
\text { y su hijo }\end{array}$ & No & No & $\begin{array}{l}\text { Producción porcina. } \\
\text { Siembra para } \\
\text { alimentar a los } \\
\text { cerdos. Aves de } \\
\text { corral }\end{array}$ & Sí \\
\hline $\begin{array}{l}2 \text { (Carhué, } \\
\text { Adolfo Alsina) }\end{array}$ & $\begin{array}{l}675 \text { has. en } \\
\text { propiedad. } 400 \\
\text { arrendamiento }\end{array}$ & $\begin{array}{l}\text { Él, su } \\
\text { hermano y } \\
\text { su padre }\end{array}$ & $\begin{array}{l}\text { Sí. } \\
\text { Su hermano } \\
\text { es contratista } \\
\text { y utilizan su } \\
\text { maquinaria }\end{array}$ & $\begin{array}{l}\text { Sí. Un } \\
\text { trabajador } \\
\text { permanente }\end{array}$ & $\begin{array}{l}\text { Producción pecuaria } \\
\text { (bovinos) y agrícola }\end{array}$ & Sí \\
\hline $\begin{array}{l}3 \text { (Darregueira, } \\
\text { Paraje La } \\
\text { Rosalía, Puan) }\end{array}$ & $\begin{array}{l}313 \text { has. (50\% } \\
\text { en propiedad } \\
\text { y } 50 \% \text { en } \\
\text { arrendamiento) }\end{array}$ & $\begin{array}{l}\text { Él, su hijo y } \\
\text { su nuera }\end{array}$ & $\begin{array}{l}\text { Sí. Toda menos } \\
\text { cosechadora } \\
\text { Cosecha: } \\
\text { contratista }\end{array}$ & No & $\begin{array}{l}\text { Producción pecuaria } \\
\text { (bovino y porcino) } \\
\text { y agrícola. Huerta } \\
\text { y producción de } \\
\text { olivos en menor } \\
\text { medida. Aves de } \\
\text { corral. }\end{array}$ & No \\
\hline $\begin{array}{l}4 \text { (Paraje } \\
\text { El Cardal, } \\
\text { Bordenave, } \\
\text { Puan) }\end{array}$ & $\begin{array}{l}330 \text { has. en } \\
\text { propiedad }\end{array}$ & $\begin{array}{l}\text { Él y su } \\
\text { esposa. } \\
\text { Un hijo } \\
\text { tiene poca } \\
\text { vinculación }\end{array}$ & $\begin{array}{l}\text { Si. No tiene } \\
\text { cosechadora }\end{array}$ & $\begin{array}{l}\text { Para momentos } \\
\text { determinados } \\
\text { llaman al hijo }\end{array}$ & $\begin{array}{l}\text { Producción pecuaria } \\
\text { (bovinos, porcinos } \\
\text { y ovinos), apícola. } \\
\text { Huerta y aves de } \\
\text { corral }\end{array}$ & No \\
\hline $\begin{array}{l}5 \text { (Villa Iris, } \\
\text { Puan) }\end{array}$ & $\begin{array}{l}1280 \text { has. en } \\
\text { propiedad. } 200 \\
\text { arrendamiento }\end{array}$ & $\begin{array}{l}\text { Él, su padre, } \\
\text { su madre y } \\
\text { su hermano }\end{array}$ & $\begin{array}{l}\text { Sí. Tractor, } \\
\text { arrolladora, } \\
\text { sembradora, } \\
\text { cosechadora y } \\
\text { rastra de disco. }\end{array}$ & $\begin{array}{l}\text { Para la cosecha } \\
\text { o casos de } \\
\text { emergencia }\end{array}$ & $\begin{array}{l}\text { Producción pecuaria } \\
\text { (bovinos), agrícola } \\
\text { (trigo, girasol y } \\
\text { maíz) }\end{array}$ & No \\
\hline
\end{tabular}

Elaboración propia a partir de la información relevada de las entrevistas realizadas

8 Integrantes de la familia vinculados a la explotación agropecuaria.

9 Utilización de maquinaria propia en la actividad agropecuaria o contratación del servicio.

10 Contratación de mano de obra (permanente o no) por fuera del núcleo familiar.

11 Desarrollo de actividades fuera del predio productivo por la que se recibe una contraprestación, ya sea en dinero o en especie. 
Persistencia de productores familiares en el sudoeste bonaerense. Un estudio de casos para su caracterización por Sofía Hang y Edgardo Gabriel Gonzalez

\section{Resultados y discusión}

\section{Análisis de las entrevistas en relación a los objetivos de la investigación}

La metodología empleada y el posterior análisis de las entrevistas realizadas, permiten abordar el objetivo principal que se plantea esta investigación: la caracterización de los productores familiares del sudoeste bonaerense.

De los cinco casos en estudio y en relación con la bibliografía consultada y desarrollada en el marco teórico, se confirma la heterogeneidad de productores que se pueden incluir dentro de lo conceptualizado como agricultura familiar.

Partiendo de la concepción de que la cantidad de tierra que se tiene en propiedad (o tenencia) que constituye la explotación agropecuaria no es un parámetro suficiente para determinar si estamos frente a un productor/a familiar, resulta necesario incorporar otras dimensiones al análisis. No obstante, se observa la disparidad de cantidad de tierra que se trabaja y en propiedad por parte de cada entrevistado, lo que demuestra que sujetos con una disparidad importante de tierra en su poder puedan ser caracterizados dentro de la agricultura familiar. Debe aclararse que dicha disparidad muestra niveles muy diferenciados de ingresos y de capital.

En relación a la propiedad de la tierra, en los cincos casos son propietarios y esta es una característica de la zona y de los sujetos en cuestión.

En los cinco casos en estudio nos encontramos con una dirección familiar y el aporte de mano de obra familiar, que es una característica típica del sector.

Los casos que contratan mano de obra asalariada por fuera del núcleo familiar lo hacen en momentos específicos del ciclo productivo, salvo un caso que tiene un trabajador permanente pero que de todas formas la dirección de la explotación continúa en la familia, como el resto de la mano de obra utilizada.

En todos los casos existe la idea de la preservación del patrimonio familiar y la continuidad de la explotación agropecuaria en manos de los hijos y nietos es tanto una preocupación como una proyección en el tiempo. Esto es una característica fundamental de la agricultura familiar que la diferencia de la organización empresarial, su permanente preocupación por la continuidad en la actividad y el mantenimiento de valores y prácticas socioproductivas.

Además, de las entrevistas se desprende que las familias viven en el campo, salvo en un caso que por cuestiones de salud viven en el pueblo, pero todos los días van al campo a desarrollar las actividades. Aquí, se puede ver reflejado cómo se unen la unidad productiva y la unidad doméstica en un mismo predio y la valoración de "estar en el campo" de manera permanente para poder realizar las actividades diversificadas que desarrollan. Esta dimensión debe entenderse 
dentro del marco de despoblamiento de zonas rurales que se da en Argentina como consecuencia de la desaparición de miles de explotaciones agropecuarias en los últimos años.

En cuanto a la pluriactividad se encuentra que en dos casos ocurre por parte de miembros del grupo familiar. Se debe tener en cuenta que esta pluriactividad se da tanto en un productor con poca cantidad de tierra, como en otro con mayor cantidad, por lo que no podemos sostener que esta situación solo se da en los la agricultura familiar menos capitalizada. Además, en uno de los casos que no tiene pluriactividad se recalca la actividad apícola como una fuente de ingreso monetario importante en la economía familiar.

La diversificación de la producción es algo muy común en la agricultura familiar que responde a diversos factores, pero que muchas veces se da como estrategia para garantizar la permanencia.

Tanto la adquisición de elementos necesarios para la producción como la comercialización de lo producido se realiza mediante intermediarios radicados en la zona, destacándose que no comercializan directamente sus producciones principales (ganadería, cereales) a frigoríficos o molinos harineros, como sí lo hacen otros productores, como los empresariales. Es indistinto para los entrevistados realizarlo mediante un consignatario privado o mediante una cooperativa agropecuaria.

Esto último puede demostrar un alejamiento de las tradiciones del sector que fueron forjadores de las entidades cooperativas, eligiendo básicamente a quien le abone más por su producción para comercializarla.

También se visualiza una característica de este sector capitalizado de la agricultura familiar, dado por la producción excedentaria destinada al autoconsumo que tiene destino extra predial.

En relación al segundo objetivo de la investigación, todos los requisitos enumerados en el art. 5 de la ley $N^{\circ}$ 27.118, se cumplen en los casos analizados, ya que la gestión del emprendimiento productivo es ejercida por las familias productoras, son propietarias de parte de los medios de producción, el trabajo lo realizan los miembros de la familia con aportes complementarios de asalariados en algunos momentos, viven en el campo o en la localidad más cercana y el ingreso económico principal es el que se desprende de la actividad agropecuaria.

Asimismo, el articulado de la ley realiza una enumeración de diversos sujetos que quedan incluidos en el concepto de agricultor y agricultora familiar. Entre ellos, enuncia a los "chacareros", figura que representa a los productores familiares típicos de la provincia de Buenos Aires, y de esta región en particular. 
Persistencia de productores familiares en el sudoeste bonaerense. Un estudio de casos para su caracterización por Sofía Hang y Edgardo Gabriel Gonzalez

\section{Permanencia en el territorio}

Todos los casos estudiados, persisten y permanecen en el territorio, a pesar de los cambios en la estructura agraria argentina, la concentración de la tierra para uso agrario en pocas manos y la desaparición de miles de explotaciones agropecuarias. Si analizamos los datos censales de 1988, 2002 y 2018 podemos observar la desaparición de más de 150.000 explotaciones agropecuarias entre 1988 y 2018 y el crecimiento promedio por explotación agropecuaria de la cantidad de hectáreas, es decir menos productores con mayor cantidad de tierra en su poder. A pesar de esta situación, estos productores han permanecido y aún permanecen en el territorio. Una de las razones de su permanencia, frente a la desaparición de sus pares en la zona núcleo pampeana, es el mantenimiento de la diversificación productiva, resaltándose la actividad ganadera extensiva como característica de este grupo de productores.

Azcuy Ameghino y Fernández (2019), sobre la cantidad de explotaciones agropecuarias de acuerdo a los datos del Censo Nacional Agropecuario que se realizó en el año 2018, explican: "Considerando la totalidad de las EAP censadas (con y sin límites definidos) el CNA 2018 registró 250.881 unidades, contra 333.533 contabilizadas en 2002, lo que implica la desaparición de 82.652, aproximadamente una cuarta parte, a un promedio de eliminación anual de 5.166 EAP. Por su parte, la superficie correspondiente a las explotaciones con límites definidos habría disminuido de 174.808.564 ha en 2002 a 157.423.932 en 2018 " (2019, p. 1).

Del estudio de las entrevistas y análisis de fuentes primarias y secundarias, se desprende que estos sujetos se han adaptado de diversas formas a estos cambios. La incorporación de tecnologías solo en la medida necesaria, la ampliación de la unidad productiva en algunos casos (compra de tierra o arrendamiento), diversas formas de asociarse vinculadas a lo impositivo (un caso de una sociedad compuesta por tres firmas), incorporación de nuevas actividades productivas y mantener la diversificación de origen familiar y de los hijos e hijas a la explotación agropecuaria, son algunos ejemplos de estrategias incorporadas para garantizar la permanencia en el territorio.

De este modo, se puede afirmar que estos sujetos han podido desarrollarse y continuar las explotaciones agropecuarias a través de la incorporación de diversas estrategias que se fueron dando a lo largo de los años, sin embargo, en sus características principales y específicas, se han mantenido.

Resulta necesario hacer hincapié en las características propias del territorio en estudio, entendiendo al sudoeste bonaerense es una zona marginal de la zona núcleo productiva del país, y la vinculación entre esas características y la permanencia en el territorio. 
Estas familias tienen un fuerte arraigo a sus explotaciones agropecuarias, es decir tienen una vinculación con el predio productivo y la localidad/ciudad cercana que los hace partícipes de la vida local. La preocupación por lo local excede lo meramente productivo.

Por este motivo, resulta sumamente interesante lo planteado por Albadalejo y Cittadini (2017) que puede aplicarse a estos casos, en cuanto a que "son vecinos, o sea, son partícipes de la «ciudad» local (del espacio público). Queda claro que este estatuto de «ciudadano», en el sentido de miembro del espacio público local, está dado no sólo por su actividad e importancia económica, sino también porque son propietarios locales" (Albadalejo \& Cittadini 2017, p. 25).

En la mayoría de los casos, se piensa y proyecta a los predios productivos desde y hacia la continuidad intergeneracional, generando estrategias productivas más amigables con los bienes naturales, observándose diferentes estrategias de combinación de diversas producciones (agrícola-ganadera-aves de corralhuerta-etc.) y rotación de los predios en producción.

Las técnicas utilizadas y la triangulación entre las mismas, dan cuenta de la relación entre las dimensiones y nudos analíticos presentados.

La bibliografía consultada presenta la heterogeneidad de los sujetos y la dificultad de su caracterización, cuestión que las entrevistas confirman. Esta relación (bibliografía y entrevistas) permite acercarse a una definición de agricultura familiar más compleja, completa e integral. Además, pone en tensión la diversidad de sujetos que se encuentra en los territorios. La normativa vinculada a la temática recoge estas cuestiones. Por otro lado, el análisis de datos de censos refleja la desaparición de productores que se confirma con lo que se observa en los territorios y se desprende de las entrevistas. La agricultura familiar desaparece, pero también persiste en el sudoeste bonaerense. Esta persistencia es el resultado de diferentes estrategias que se ven en las entrevistas y es una característica propia de estos sujetos. 


\section{Reflexiones finales}

La bibliografía consultada, el análisis de las entrevistas y la normativa vigente reflejan la heterogeneidad de sujetos productivos que habitan los territorios y en este caso en particular el sudoeste de la provincia de Buenos Aires, que mantiene un alto número de explotaciones agropecuarias familiares, a pesar de la gran cantidad de EAPs desaparecidas en las últimas décadas.

Los sujetos analizados quedan incluidos en las diversas conceptualizaciones que se hacen sobre la agricultura familiar. Esto genera que tengan ciertas características propias, no sólo en lo productivo, sino también en su vinculación con la explotación agropecuaria, el medio local que habitan y los vínculos intrafamiliares.

La caracterización y la conceptualización de estos sujetos, resulta imprescindible para la formulación y ejecución de políticas públicas que sean pensadas e ideadas direccionadas a los sujetos. Por este motivo, considerarlos como productores familiares no es sólo un ejercicio teórico sino una necesidad para pensar los abordajes territoriales desde el estado. 


\section{Referencias bibliográficas}

Albadalejo, A., \& Cittadini, R. (2017). El productor silencioso: destino del gran actor de la modernización de los años 1960-70 en la actual copresencia de agriculturas de la región pampeana argentina. PAMPA, (16), 9-34. https://doi.org/10.14409/pampa.v0i16.6949

Azcuy Ameghino, E., \& Martínez Dougnac. G. (2009). La agricultura familiar pampeana entre la realidad y el mito. XXVII Congreso de la Asociación Latinoamericana de Sociología. VIII Jornadas de Sociología de la Universidad de Buenos Aires. Asociación Latinoamericana de Sociología. Congreso llevado a cabo en la ciudad de Buenos Aires. Disponible en: http://cdsa.aacademica. org/000-062/397

Azcuy Ameghino, E., \& Fernández, D. (2019). El Censo Nacional Agropecuario 2018: visión general y aproximación a la región pampeana. Centro Interdisciplinario de Estudios Agrarios, Buenos Aires: Universidad de Buenos Aires. Disponible en: https://bichosdecampo.com/wp-content/uploads/2019/12/cna_2018_ azcuy_ameghino_fernandez-1.pdf

Balsa, J., \& López Castro, N. (2011). La agricultura familiar ‘moderna'. Caracterización y complejidad en sus formas concretas en la región pampeana". En N. López Castro, \& G. Prividera. (Comps.), Repensar la agricultura familiar. Aportes para desentrañar la complejidad agraria pampeana (pp.45-75). Buenos Aires: CICCUS.

Balsa, J. (2012). Agricultura familiar: caracterización, defensa y viabilidad. Revista Interdisciplinaria de Estudios Agrarios, 36, 5-28. Disponible en: http:// bibliotecadigital.econ.uba.ar/download/riea/riea_v36_n1_01.pdf

Benavides, M. O., \& Gómez Restrepo, C. (2005). Métodos en investigación cualitativa: triangulación. Revista Colombiana de Psiquiatría, 34(1), 118124. Disponible en: http://www.scielo.org.co/scielo.php?pid=S0034$74502005000100008 \&$ script $=$ sci_abstract\&tlng=es

Bustos Cara, R. (2010) La perspectiva territorial en el desarrollo rural en el sudoeste de la provincia de Buenos Aires. Anales de la ANAV. Trabajos del tomo LXIV, 323-336. Bahía Blanca, Argentina: ANAV.

FONAF (2006). Documento de los lineamientos generales de políticas públicas orientadas a la elaboración de un plan estratégico para la agricultura familiar. Disponible en: http://fediap.com.ar/administracion/pdfs/Lineamientos\%20 para\%20Pol\%C3\%ADticas\%20P\%C3\%BAblicas\%20orientada\%20a\%20 la\%20Elaboraci\%C3\%B3n\%20de\%20un\%20Plan\%20Estrat\%C3\%A9gico\%20 para\%20la\%20Agricultura\%20Familiar\%20-\%2006.pdf 
Ley Nacional N²7.118. Boletín Oficial de la República de Argentina. 20 de enero de 2015. Buenos Aires, Argentina.

López Castro, N. (2010). Cuando la persistencia es una cuestión de familia. Relaciones familiares, traspaso y género en explotaciones agropecuarias del Sudoeste bonaerense (1987-2007). Mundo Agrario, 10(19). Disponible en: http://www.mundoagrario.unlp.edu.ar/article/view/v10n1

López Castro, N. (2012). Persistencia en los márgenes. La Agricultura Familiar en el sudoeste Bonaerense. Buenos Aires: Fundación CICCUS.

López Castro, N. (2013). Transformaciones sociales y procesos de diferenciación social de la producción familiar pampeana. Estudio sobre el agro del sudoeste bonaerense en las últimas décadas (Puán y Adolfo Alsina, 1988-2012). (Tesis de Doctorado). Universidad Nacional de Quilmes, Bernal, Argentina. Disponible en: http://ridaa.unq.edu.ar/handle/20.500.11807/126

López Castro, N. (2013). La producción familiar en el SO bonaerense de las últimas décadas: claves productivas de su persistencia (Puán y Adolfo Alsina, 19882012). Huellas (17), 187-203. Disponible en: http://cerac.unlpam.edu.ar/ index.php/huellas/article/view/858

Martínez Carazo, P. (2006). El método de estudio de caso: estrategia metodológica de la investigación científica. Pensamiento \& Gestión, (20), 165-193. Disponible en: https://www.redalyc.org/pdf/646/64602005.pdf

Martinez Dougnac, G., \& Azcuy Ameghino, E. (2014). La Agricultura Familiar Pampeana: Notas Sobre Historia y Actualidad. Eutopía. Revista De Desarrollo Económico Territorial, (6), 41-52. https://doi.org/10.17141/ eutopia.6.2014.1401

Soverna, S., Tsakoumagkos, P., \& Paz, R. (2008). Revisando la definición de agricultura familiar. Buenos Aires: Secretaría Agricultura, Ganadería, Pesca y Alimentos. Disponible en: https://aulavirtual.agro.unlp.edu.ar/pluginfile. php/13509/mod_resource/content/1/2012/Soberna_et_al_Revisando_la_ definicioin_de_agricultura_familiar.pdf

Dirección de correspondencia:

Sofía Hang

Contacto: sofihang@hotmail.com

Esta obra se encuentra bajo una Licencia de Creative Commons

Reconocimiento-NoComercial-Compartirlgual 4.0 Internacional 